Europhys. Lett., 21 (4), pp. 439-444 (1993)

\title{
Proposal for a Magneto-optical Beam Splitter for Atoms.
}

\author{
T. PfaU, C. S. Adams and J. Mlynek \\ Fakultät für Physik, Universität Konstanz - D-7750 Konstanz, Germany
}

(received 10 August 1992; accepted in final form 23 November 1992)

PACS. 32.80 - Photon interactions with atoms.

PACS. 42.50 - Quantum optics.

\begin{abstract}
In this letter we present a theoretical study of the coherent diffraction of three-level atoms from a light field with a polarization gradient (counterpropagating crossed linearly polarized beams) and a static magnetic field applied parallel to the laser propagation direction. We show that for a particular ratio of the laser field intensity and the magnetic-field strength, there occurs a resonance between the Larmor precession of the magnetic alignment and the Rabi oscillations. On resonance the atomic wave function is diffracted by an approximately triangular optical potential which leads to a very efficient coherent splitting of the atomic beam. The proposed configuration is particularly interesting in relation to atom interferometry, when efficient coherent beam splitters for atoms are required.
\end{abstract}

Introduction. - Atom interferometry has a considerable potential both as a technique for precision measurement and as a means to perform fundamental tests of our understanding of quantum theory [1]. For many applications the sensitivity of an interferometer is proportional to the area enclosed by the two paths. Consequently, an important consideration in atom interferometry is a large spatial splitting of the atomic wave function. In previous experiments coherent atomic-beam splitters were realized by diffraction from microstructures [2,3] or an optical standing wave [4], $\pi / 2$ laser pulses [5], stimulated Raman transitions [6] and the optical Stern-Gerlach effect [7].

For microstructures the splitting is inversely proportional to the grating period and therefore limited by microfabrication techniques. The momentum distribution produced by coherent diffraction from a standing light wave has an envelope given by a Bessel function distribution. For this reason, standing-wave diffraction is not an efficient technique for producing a coherent splitting into high-transverse-momentum states. In the case of single-photon excitation or stimulated Raman transitions, the maximum momentum kick imparted to the atom per process is limited to one or two photon momenta $(\hbar k)$, respectively. A larger splitting of order 8$k k$ was achieved using the optical Stern-Gerlach effect [7]. Another proposed method for an effective beam splitting is based on the adiabatic passage between Zeeman sublevels for multilevel atoms [8].

In this paper we report on a new approach to coherently split an atomic beam. We consider the diffraction of atoms in a polarization gradient light field (counterpropagating crossed linearly polarized beams) and a static magnetic field applied parallel to the laser propagation direction (fig. 1a)). For a particular ratio of the laser intensity and magnetic-field strength, one of the eigenstates of the interaction experiences an approximately triangular potential in the transverse direction resulting in a large, clearly two-peaked splitting in momentum space. The total splitting is proportional to the light- and 


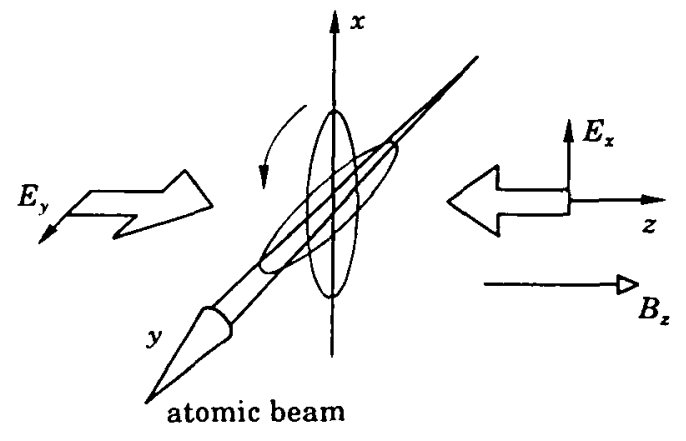

a)

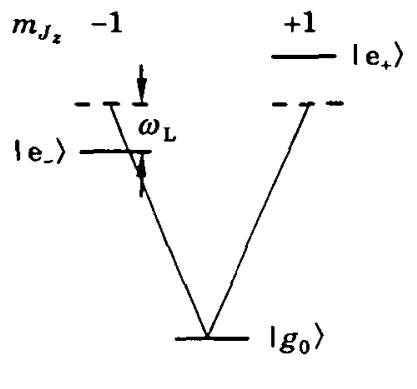

b)

Fig. 1. $-a$ ) The proposed configuration of the laser fields $E_{x}$ and $E_{y}$, and the magnetic field $B_{z}$, relative to the atomic-beam direction $(y)$. In this simplified picture the momentum transfer process is explained by the Larmor precession of the excited-state magnetic alignment (represented by orthogonal ellipsoides) and the coupling of the two beams to the orthogonal states of the alignment. $b$ ) The level scheme for a $J=0$ to $J^{\prime}=1$ transition with the quantization axis defined parallel to the magnetic field, ie. along $z$.

magnetic-field amplitudes. As the splitting process is adiabatic (see sect. 3), the two outgoing parts of the wave function are both in the ground state, i.e. the process is nonpolarizing. This is ideal for applications in atom interferometry.

The physical mechanism of the beam-splitting effect is analogous to the magneto-optical force proposed and demonstrated by Grimm et al. [9]. For the coherent process, i.e. no spontaneous emission, and by preserving the symmetry using crossed linearly polarized beams, the magneto-optical interaction produces a symmetric beam splitting. A simple picture can be used to understand the process intuitively: the atomic level scheme is displayed in fig. $1 b$ ) for a choice of the quantization axis parallel to the magnetic field, i.e. along the $z$-axis. In this situation linearly polarized light couples the ground state to a linear superposition of the excited-state levels and induces a so-called alignment of the atom. The perpendicular linearly polarized light couples the ground state to the orthogonal alignment. Both alignments are represented by ellipsoides in fig. 1a). A magnetic field induces a precession of an alignment. This Larmor precession changes the probability of absorbing or emitting photons from one direction or the other. If the frequency of the absorption and emission cycles is twice the Larmor precession frequency there is a resonance, and the atom repeats cycles of absorption from beam 1 and emission into beam 2 or vice versa. In this simplified picture, the phase of the absorption and the emission processes on resonance is preserved and an effective beam splitting is predicted.

The picture described is an oversimplification as the alignment does not evolve freely, ie. the atomic system is strongly coupled both to the magnetic and to the light field. In other words, the system cannot be treated in a perturbative way.

Dressed-state model. - A physical insight into the role of the magnetic and laser fields is provided by the nonperturbative treatment considering the dressed states of the interaction in the position representation. The following discussion is applicable to any transition containing a symmetric $\vee$ or $\wedge$ level scheme. We consider a $J=0 \rightarrow J=1$ transition and choose the quantization axis parallel to the magnetic-field direction, i.e. along $z$ (fig. 1a)). In 
this basis the $\left|e_{0}\right\rangle$ sublevel does not interact with the laser or the magnetic field and the level scheme reduces to a $V$ system as shown in fig. $1 b$ ).

The wave function of the atom in the position representation may be written as

$$
|\Psi\rangle=\sum_{i} \int \psi_{i}(\boldsymbol{r}, t)|i\rangle|\boldsymbol{r}\rangle \mathrm{d} \boldsymbol{r},
$$

where $i=\left\{g_{0}, e_{-}, e_{+}\right\}$is the internal state and $r$ is the centre-of-mass position. The magnetic interaction is $H_{\mathrm{B}}=-\boldsymbol{\mu} \cdot \boldsymbol{B}$, where $\boldsymbol{\mu}=-g_{J} \mu_{\mathrm{B}} J$ is the magnetic moment. The atom-laser interaction in the electric-dipole approximation is given by

$$
H_{\text {A-L }}=-\boldsymbol{d} \operatorname{Re}\{\boldsymbol{E}(\boldsymbol{r}) \exp [-i \omega t]\},
$$

where $\boldsymbol{d}$ is the electric-dipole operator and the laser field is

$$
\boldsymbol{E}(\boldsymbol{r})=E_{0}(x, y)\left(\varepsilon_{x} \exp [-i k z]+\varepsilon_{y} \exp [i k z]\right) \text {. }
$$

The atomic beam propagates in the $y$-direction. The spatial dependence of $E_{0}(x, y)$ along $y$, in the moving frame of the atom, can be written as an explicit time-dependence $t=y / u$ where $u$ is the velocity of the atom. For simplicity, we assume that the field is uniform along $x$. The linear polarization vectors are rewritten in terms of their $\sigma_{+}$and $\sigma_{-}$components, i.e.

$$
\varepsilon_{x}=\frac{1}{\sqrt{2}}\left(\varepsilon_{+}+\varepsilon_{-}\right) \quad \text { and } \quad \varepsilon_{y}=\frac{1}{\sqrt{2}}\left(\varepsilon_{+}-\varepsilon_{-}\right)
$$

Thus, in the rotating-wave approximation and for zero laser detuning (i.e. the laser frequency equal to the unperturbed transition frequency), the Hamiltonian in the interaction representation is

$$
\begin{aligned}
& H_{\text {int }}= \\
& =\hbar\left[\begin{array}{ccc}
0 & \frac{\omega_{\mathrm{R}}(t)}{2 \sqrt{2}}(\exp [i k z]+i \exp [-i k z]) & \frac{\omega_{\mathrm{R}}(t)}{2 \sqrt{2}}(\exp [i k z]-i \exp [-i k z]) \\
\frac{\omega_{\mathrm{R}}^{*}(t)}{2 \sqrt{2}}(\exp [-i k z]-i \exp [i k z]) & -\omega_{\mathrm{L}} & 0 \\
\frac{\omega_{\mathrm{R}}^{*}(t)}{2 \sqrt{2}}(\exp [-i k z]+i \exp [i k z]) & 0 & \omega_{\mathrm{L}}
\end{array}\right],
\end{aligned}
$$

where the Rabi frequency $\omega_{\mathrm{R}}(t)$ is defined as $\left({ }^{1}\right)$

$$
\omega_{\mathrm{R}}(t)=-\frac{\left\langle e_{ \pm}\left|\boldsymbol{d} \cdot \boldsymbol{\varepsilon}_{ \pm}\right| g_{0}\right\rangle}{\hbar} E_{0}(u t)
$$

and $\hbar \omega_{\mathrm{L}}=m_{J} g_{J} \mu_{\mathrm{B}}$ is the Zeeman splitting.

The eigenvalues of the interaction Hamiltonian are given by

$$
\lambda_{j}=2\left[\frac{1}{3}\left(\omega_{\mathrm{L}}^{2}+\frac{1}{2} \omega_{\mathrm{R}}^{2}\right)\right]^{1 / 2} \cos \left\{\frac{1}{3} \cos ^{-1}\left[\frac{\omega_{\mathrm{L}} \omega_{\mathrm{R}}^{2} \sin 2 k z}{4\left[\frac{1}{3}\left(\omega_{\mathrm{L}}^{2}+\frac{1}{2} \omega_{\mathrm{R}}^{2}\right)\right]^{1 / 2}}\right]+\frac{2 \pi}{3} j\right\},
$$

where $j=1,2$ and 3 . In fig. 2 the eigenvalues are plotted as a function of the position in the polarization gradient for three values of the laser intensity. For $\omega_{R}=0.5 \omega_{L}$ or $\omega_{R}=4.5 \omega_{L}$

(1) Note that $\omega_{R}$ is the Rabi frequency for one laser beam. 
(fig. $2 a$ ) and $c$ )), there are three separate, spatially modulated eigenvalues. In a weak laser field (fig. 2a)), the eigenstates $|1\rangle,|2\rangle$ and $|3\rangle$ correspond approximately to the magnetic eigenstates, i.e. to the initial states $\left|e_{-}\right\rangle,\left|g_{0}\right\rangle$ and $\left|e_{+}\right\rangle$, respectively. The separation is equal to the Zeeman splitting of the excited state and the spatial modulation is due to the light shift, which is proportional to the intensity of the $\sigma_{+}$or $\sigma_{-}$field component. In the strong-laser-field (or weak-magnetic-field) case, the eigenstates correspond to mixtures of the magnetic sublevels (fig. 2c)). In the limit $\omega_{\mathrm{R}} \gg \omega_{\mathrm{L}}$ the light shift of the eigenstates becomes independent of the position.

For $\omega_{\mathrm{R}}=2 \omega_{\mathrm{L}}$ the light shift on one transition at the positions of pure $\sigma_{+}$or $\sigma_{-}$light is sufficient to bring the other transition exactly to resonance and the level anticrossing shows a degeneracy (fig. $2 b)$ ). In this case, the first-order perturbation induced by a small $\sigma_{-}$or $\sigma_{+}$ component does not vanish (in contrast to the nondegenerate case). Thus, due to the linear increase of the perturbation on either side of the anticrossing, the levels are perturbed

a)
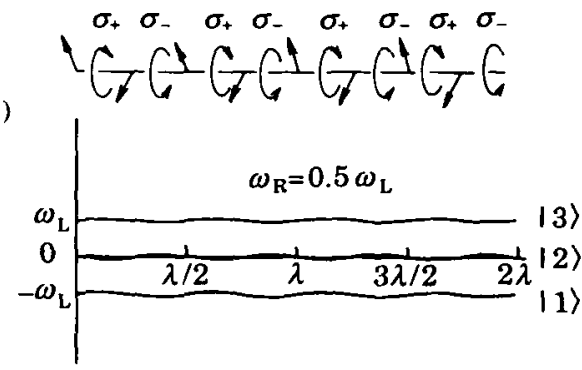

b)

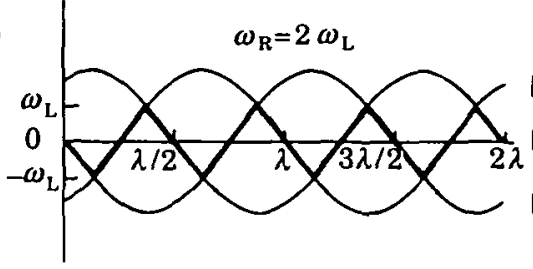

c)

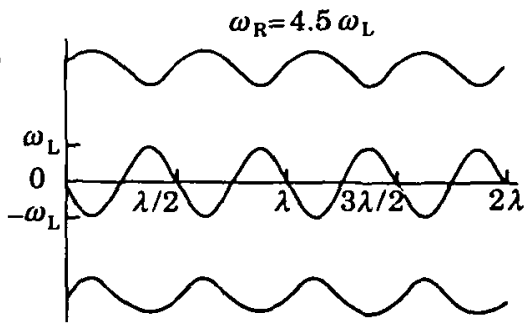

Fig. 2.

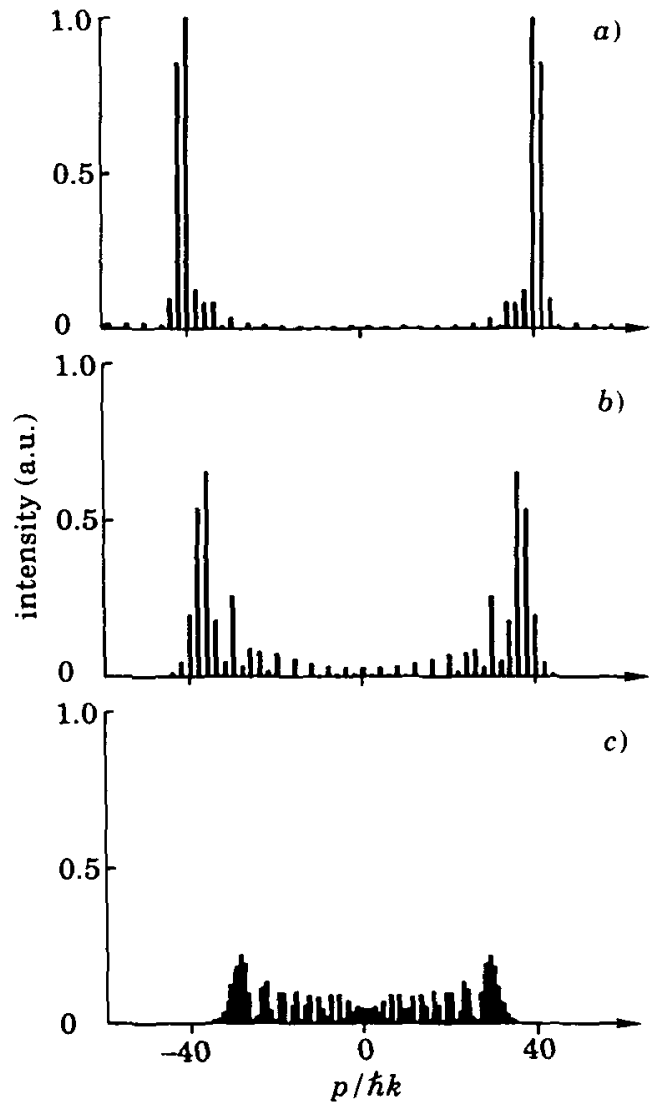

Fig. 3.

Fig. 2. - The spatial dependence of the eigenvalues of the interaction Hamiltonian for various ratios of the Rabi frequency $\omega_{R}$ and the Larmor frequency $\left.\left.\omega_{L}: a\right) \omega_{R}=0.5 \omega_{L}, b\right) \omega_{R}=2 \omega_{L}$ and $c$ ) $\omega_{R}=4.5 \omega_{L}$.

Fig. 3. - The momentum distribution for a three-level atom diffracted by a polarization gradient light field with a static magnetic field in the adiabatic limit after $t=10^{*} 2 \pi / \omega_{\mathrm{R}}$ for: $a$ ) a uniform laser field, $b$ ) a Gaussian laser field. c) For comparison the momentum distribution produced by diffraction of a two-level atom from a standing-wave light field. 
linearly along $z$, leading to a discontinuity in the potential gradient at positions of pure $\sigma_{+}$or $\sigma_{-}$polarizations (fig. $\left.2 b\right)$ ). The central eigenstate $|2\rangle$ is responsible for the beam-splitting effect. An atom in this state sees an optical potential with an approximately triangular spatial dependence shown by the thick line in fig. $2 b$ ). The potential divides the wave function into two parts which experience opposite forces. The magnitude of the force is given by the potential gradient. It can be seen from fig. $2 b$ ) that the gradient is approximately equal to the Zeeman splitting $2 \hbar \omega_{\mathrm{L}}$ divided by $\lambda / 4$. Thus the net force is $8 \hbar k \omega_{\mathrm{L}} / 2 \pi$ (ie. $\left.4 \hbar k \omega_{\mathrm{R}} / 2 \pi, \operatorname{see}\left({ }^{1}\right)\right)$.

The eigenvalues of the other states display a more sinusoidal spatial dependence and give rise to a diffraction similar to that produced by a normal standing wave. It follows that in order to observe an efficient beam splitter, we would like to prepare the atom in eigenstate $|2\rangle$.

Adiabatic limit. - In an experiment where the interaction time is greater than a few Rabi cycles (which we require in order to achieve a significant splitting) the switching times of the interaction are slow compared to the characteristic time scale of the magneto-optical process $\left(\omega_{\mathrm{L}}\right.$ or $\left.\omega_{\mathrm{R}}\right)$. In this case the ground state $\left|g_{0}\right\rangle$ evolves predominantly adiabatically into eigenstate $|2\rangle[10]$. In the neighbourhood of the level anticrossings there is always a small region of nonadiabatic behaviour. By integrating the Schrödinger equation, we found that for parameters leading to a large splitting (discussed in sect. 4), the fraction of atoms undergoing nonadiabatic transitions was less than a few percent. Therefore for further calculations we assume perfect adiabatic evolution.

The momentum distribution in the adiabatic limit was calculated by considering the eigenvalue potential as a phase grating (i.e. the momentum distribution is given by the Fourier transform of $\exp \left[i \int \lambda_{2}(z, t) \mathrm{d} t\right]$, where $\lambda_{2}$ is the triangular eigenvalue). The calculation assumes that the change in the kinetic energy of the atom induced by the interaction is negligible (this is known as the Raman-Nath approximation). In the position representation the Raman-Nath regime can be interpreted as the limit where the transverse displacement of the atom during the interaction is small compared to the wavelength of the optical potential. The Raman-Nath assumption places an upper limit on the maximum momentum splitting we are able to predict using our model. Note that the Raman-Nath limit is specific to a particular atomic system.

Calculations in the adiabatic limit and discussion. - In the following example the calculations were made using parameters corresponding to a supersonic helium beam and a light field resonant with the $2^{3} S_{1}$ to $2{ }^{3} P_{1}$ transition. We assume that the atom is optically pumped in the $m_{J}=0$ ground-state level. In order to remain in the Raman-Nath regime we limit the maximum transverse displacement to one tenth of the period of the optical potential.

Initially, we assumed a square profile interaction where the switching times are much shorter than the total interaction time, but sufficiently long to allow adiabatic evolution. The calculated momentum distribution is shown in fig. $3 a$ ). In this example we observe an extremely efficient beam splitter with a momentum splitting of $\pm 40 \hbar k$. There is a small amount of scattering into adjacent momentum states because the potential is not perfectly linear but part of a sine wave, and due to the finite width of the linear regions. The splitting process is relatively insensitive to the laser detuning. The effect is not significantly disturbed until the detuning becomes comparable to the Rabi frequency.

The square profile interaction discussed above is difficult to realize experimentally. As we are interested in the coherent process, it is necessary to avoid spontaneous emission. The splitting process is most efficient with no laser detuning, therefore the preferred technique for achieving a coherent interaction is to arrange that the interaction time is much shorter than the excited-state lifetime. This can easily be achieved using a supersonic beam of 
metastable helium atoms by focussing the laser beam, as demonstrated in the experimental observation of the optical Stern-Gerlach effect [7]. However, the small laser spot size makes it difficult to match the Rabi frequency and the Larmor frequency throughout the interaction. An experimentally more realistic configuration consists of a Gaussian profiled light field and an approximately uniform magnetic field. The expected momentum distribution for this case, again in the adiabatic limit, is shown in fig. $3 b$ ). As the resonance condition is not maintained throughout the interaction, there is a slight increase in the momentum spread of the split beams.

In fig. $3 c$ ) we show for comparison the diffraction pattern from a normal standing wave with zero laser detuning, and a laser intensity corresponding to the same potential height as above. The envelope of the diffraction pattern is the square of a Bessel function. Thus after many Rabi periods there is a broad spread in momentum rather than a clear splitting into high-order momentum states. In contrast the magneto-optical interaction produces a clearly two-peaked envelope, i.e. it leads to an efficient beam splitting into high-order momentum states.

The effects of non-adiabatic transitions, the solution for arbitrary angles between the polarizations and the calculations for a $J=1$ to $J^{\prime}=2$ transition will be published elsewhere.

Conclusion. - In this letter we have presented theoretical simulations of the coherent scattering of three-level atoms in a light field formed by perpendicular linearly polarized running waves. The excited state of the atom is split by a magnetic field applied parallel to the laser propagation direction. For a particular ratio of the laser intensity and the magnetic-field strength, there occurs a resonant-scattering effect due to a phase relationship between the absorption and stimulated emission cycles and the mixing of the excited-state Zeeman levels. For experimental parameters applicable to helium we predict a splitting of $\pm 40 \hbar k$. The maximum splitting angle is limited by the available laser power or magnetic-field strength. A large coherent splitting of the atomic wave function may be used as a coherent-beam splitter in an atom interferometer.

We would like to thank T. SLEATOR for many helpful suggestions, in particular concerning the adiabatic behaviour, and also C. CoHEn-TANNoudJ, R. GRIMM and CH. KURTSIEFER for fruitful discussions. CSA gratefully acknowledges the support of the Royal Society's European Science Exchange Programme. This work is supported by the Deutsche Forschungsgemeinschaft.

\section{REFERENCES}

[1] Optics and Interferometry with Atoms (special issue), Appl. Phys. B, 54 (1992) 319-485.

[2] Carnal, O. and Mlynek J., Phys. Rev. Lett., 66 (1991) 2689.

[3] Keith D. W., Ekstrom C. R., Turchette Q. A. and Pritchard D. E., Phys. Rev. Lett., 66 (1991) 2693.

[4] Gould P. L., Martin P. J., Ruff G. A., Stoner R. E., Picque J.-L. and Pritchard D. E., Phys. Rev. $A, 43$ (1991) 585.

[5] Riehle F., Kisters Th., Witte A., Helmcke J. and Bordé C. J., Phys. Rev. Lett., 67 (1991) 177.

[6] Kasevich M. and ChU S., Phys. Rev. Lett., 67 (1991) 181.

[7] Sleator T., Pfau T., Balykin V., Carnal O. and Mlynek J., Phys. Rev. Lett., 68 (1992) 1996.

[8] Marte P., Zoller P. and Hall J. L., Phys. Rev. A, 44 (1991) 4118.

[9] Grimm R., Ovchinnikov Yu. B., Sidorov A. I. and Letokhov V. S., Opt. Commun., 84 (1991) 18; Grimm R., Letokhov V. S., OvCHINNIKov YU. B. and Sidorov A. I., JETP Lett, 54 (1991) 615; J. Phys. II, 2 (1992) 93.

[10] Sleator T., private communication. 